\title{
IS CONSCIOUSNESS A GRADUAL PHENOMENON? \\ EVIDENCE FOR AN ALL-OR-NONE BIFURCATION DURING \\ THE ATTENTIONAL BLINK.
}

Claire Sergent and Stanislas Dehaene

Institut National de la Santé et de la Recherche Médicale Unité 562,

Service Hospitalier Frédéric Joliot, Commissariat à l'énergie Atomique, Orsay, France

Corresponding author : Claire Sergent

Address : INSERM U 562, Service Hospitalier Frédéric Joliot, CEA/DRM/DSV

4, place du Général Leclerc, 91401 Orsay Cedex, FRANCE

phone : 0033 (0)1 69867840

fax : 0169867816

email:sergent@shfj.cea.fr

Abstract : 122 words

Main text : 3961 words 


\begin{abstract}
Several theories of the neural correlates of consciousness assume that there is a continuum of perception, associated with a gradual change in the intensity of brain activation. But some models, considering reverberation of neural activity as necessary for conscious perception, predict a sharp non-linear transition between unconscious and conscious processing. We asked participants to evaluate the visibility of target words on a continuous scale during the attentional blink, which is known to impede explicit reports. Participants used this continuous scale in an all-or-none fashion : targets were either identified as well as targets presented outside the blink period or not detected at all. We suggest that a stochastic non-linear bifurcation in neural activity underlies the all-or-none perception observed during the attentional blink.
\end{abstract}




\section{INTRODUCTION}

Whether there can be a strict dissociation between conscious and unconscious processing is a debated issue. Some imaging studies of visual perception show a gradual increase in the cortical activity evoked by a stimulus as participants report increased knowledge of the stimulus (Bar et al., 2001; Grill-Spector, Kushnir, Hendler, \& Malach, 2000; Moutoussis \& Zeki, 2002). For example, Bar et al. (Bar et al., 2001), in a study on object recognition, observed a progressive increase in brain activation in several areas of the anterior fusiform gyrus as recognition level increased. According to Farah "consciousness may be associated only with the higher-quality end of the continuum of degrees of representation" (Farah, 2000). As noted by Kanwisher (Kanwisher, 2001), signal detection theory and connectionist models have contributed to promote the idea that mental representations are graded rather than discrete.

Other studies, however, challenge this view by showing large all-or-none changes in neural activity when a stimulus fails to be reported as compared to when it is reported (Dehaene et al., 2001; Lamme, Super, Landman, Roelfsema, \& Spekreijse, 2000; Super, Spekreijse, \& Lamme, 2001). Indeed, a qualitative difference between unconscious and conscious processing is generally expected by theories that view recurrent interactions between distant brain areas as a necessary condition for conscious perception (Dehaene, Kerszberg, \& Changeux, 1998; Dehaene \& Naccache, 2001; Di Lollo, Enns, \& Rensink, 2000; Lamme, 2003; Lamme \& Roelfsema, 2000). One of these theories (Dehaene et al., 1998) has proposed that consciousness is associated with the interconnection of multiple areas processing a stimulus by a "neuronal workspace" (B.J Baars, 1989; B. J. Baars, 1997) within which recurrent connections allow long-distance communication and auto-amplification of the activation. Neuronal network simulations (Dehaene, Sergent, \& Changeux, 2003) suggest the 
existence of a fluctuating dynamical threshold. If the primary activation evoked by a stimulus exceeds this threshold, reverberation takes place and stimulus information gains access, through the workspace, to a broad range of areas allowing, among other processes, verbal report, voluntary manipulation, voluntary action and long-term memorization. Below this threshold, however, stimulus information remains unavailable to these processes. Thus, the global neuronal workspace theory predicts an all-or-none transition between conscious and unconscious perception (see Fig. 1). More generally, many non-linear dynamical systems with self-amplification are characterized by the presence of discontinuous transitions in internal state, or "catastrophes" (Thom, 1972).

Here, we test the all-or-none character of conscious perception using an attentional blink paradigm. When two targets are embedded in a rapid sequence of distractors, correct identification of the first target (T1) hinders explicit report of the second target (T2) if they are separated by 200 to $500 \mathrm{~ms}$ (Broadbent \& Broadbent, 1987). This phenomenon is called the attentional blink (Raymond, Shapiro, \& Arnell, 1992) or AB. The AB affects a vast range of explicit tasks on $\mathrm{T} 2$, but the behavioral measures currently used to detect the $\mathrm{AB}$ (accuracy on a forced choice task), do not allow to decide whether participants are really unconscious of the blinked target, especially since accuracy is often slightly above chance level. We examined whether the $\mathrm{AB}$ merely degrades the available information on $\mathrm{T} 2$ or whether it corresponds to an all-or-none loss of conscious perception of $\mathrm{T} 2$. To this end, we asked participants to rate the visibility of $\mathrm{T} 2$ on a continuous scale. Using a continuous measure instead of discrete response categories (Bar et al., 2001) allowed us to test the continuous or discontinuous character of perceptual transitions (Massaro \& Cohen, 1983). To rule out the possibility that information on $\mathrm{T} 2$ had been forgotten by the time the question was asked, the subjective response was required immediately after the presentation of $\mathrm{T} 2$. 


\section{EXPERIMENT 1 : ALL-OR-NONE RESPONDING IN AN AB \\ PARADIGM.}

We first studied the use of the continuous scale in a classical AB paradigm in which the SOA or lag between $\mathrm{T} 1$ and $\mathrm{T} 2$ was varied. If the $\mathrm{AB}$ merely degrades the available information on $\mathrm{T} 2$, the response distribution should shift gradually from very low visibility when the $\mathrm{AB}$ is strongest (around $300 \mathrm{~ms} \mathrm{SOA}$, corresponding to lag 3 in our experiment) to higher visibility as lag increases. However, if the AB reflects increased probability of an allor-none loss of conscious perception, we predict that, for the same visual stimulation, we should be able to distinguish two types of trials : "not seen" trials where T2 completely fails to be consciously perceived, and "seen" trials where T2 is fully perceived. Thus, the distribution of perception ratings should be bimodal. Furthermore, T2 visibility in the "seen trials" should be unaffected by the T1-T2 lag. Instead, the lag should affect the relative proportions of "seen" and "not seen" trials.

\section{Methods}

\section{Subjects}

Ten right-handed native French speakers ( 5 women and 5 men; age ranging from 20 to 25) participated to Experiment 1. All had normal or corrected-to-normal vision.

\section{Procedure}

In all three experiments, participants were asked to evaluate the subjective visibility of a target (number word) on a continuous scale very shortly after target presentation (at most $300 \mathrm{~ms}$ after target onset). This scale was materialized by a horizontal bar $\left(26^{\circ} \times 2^{\circ}\right)$, 
presented at the center of the screen, labeled "not seen" at left and "maximal visibility" at right. Participants moved a cursor on the scale by pressing two designated keys on the computer keyboard, then validated their choice by pressing the space bar. The cursor, a vertical rectangle $\left(1.2^{\circ} \times 2^{\circ}\right)$, could take 21 contiguous positions on the scale by steps of $5 \%$ visibility. Its initial position was random. At the beginning of the experiment, participants were instructed to use the scale to rate the visibility of the target as finely as possible without time pressure.

\section{Design and stimuli}

In all three experiments, the stimuli were presented on a black background at the center of the computer screen $(70 \mathrm{~Hz}$ refresh) using Expe6 software (Pallier, Dupoux, \& Jeannin, 1997). The target for subjective visibility judgment was a 4-letter number word, "DEUX", "CINQ", "SEPT" or "HUIT" $\left(4^{\circ} \times 1^{\circ}\right)$, that could be present or absent. In the targetabsent condition, an empty screen was presented instead of a number word.

In this experiment, the target (T2) was embedded in a RSVP sequence of 4-uppercaseconsonant-strings distractors randomly generated using all consonants except Q,T and $\mathrm{X}$. The distractors were presented in the same font and at the same spatial location as $\mathrm{T} 2$ with the same dimensions. All items in the RSVP sequence were presented for $43 \mathrm{~ms}$, and were separated by blanks lasting $43 \mathrm{~ms}$. They were presented in blue except T1 which was white. T1 was either "XOOX" or "OXXO" with the same probability. It was either the $7^{\text {th }}$ or the $10^{\text {th }}$ stimulus in the RSVP sequence. T2 was presented either at lag 1, 2, 3, 4, 6 or 8 after T1 (corresponding to SOAs of $86,172,258,344,516$ or $688 \mathrm{~ms}$ respectively). To prevent the saliency of T2-absent trials, on every trial each distractor could be replaced by an empty screen with a $20 \%$ probability, except for the distractors surrounding $\mathrm{T} 1$ or $\mathrm{T} 2$ and the last distractor in the sequence. The subjective visibility scale was presented immediately after the 
offset of the second distracter following T2, hence $215 \mathrm{~ms}$ after T2 onset. In the "dual-task" condition, once the participant had evaluated T2 visibility, they reported whether the central letters of T1 were "OO" (T1 = "XOOX") or "XX" (T1 = "OXXO"). After a short training, each participant performed 32 trials per lag $\times$ presence condition in a dual-task session and 16 trials per lag $\times$ presence condition in a single-task session. The order of the task conditions was counterbalanced across participants.

\section{Results}

Trials with an incorrect response to $\mathrm{T} 1$ ( $2 \%$ to $16 \%$ across participants) were discarded. Mean T2 visibility rating in the different conditions followed a classical AB pattern (see Fig. 2b). An Analysis of Variance (ANOVA) restricted to the T2-present condition revealed a significant main effect of task $\left(F(1,9)=21.33, p<.001, \eta^{2}=.70\right)$ and a significant task $\times$ lag interaction $\left(F(5,45)=4.24, p<.005, \eta^{2}=.32\right)$. In the critical condition (T2-present, dual-task), mean T2 visibility decreased from lag 1 (73\%) to lag $3(51.5 \%)$, and then increased again to reach $80 \%$ at lag 8 , resulting in a significant effect of lag $(F(5,45)=10.7$, $\left.p<.0001, \eta^{2}=.54\right)$. However, when no task on T1 was required, mean visibility was higher at all lags and was little affected by lag $\left(F(5,45)=2.7, p=.03, \eta^{2}=.23\right)$. Finally, when T2 was absent, mean subjective visibility was very low (7\%) and unaffected by lag, task or their interaction $\left(F_{\mathrm{S}}<2\right)$.

We then plotted response distributions in each condition and analyzed them with ANOVAs with factors of visibility (21 levels), lag ( 7 levels), and task (2 levels). When T2 was absent, distributions showed a single peak at $0 \%$ visibility (over $60 \%$ of the responses). Conversely, when $\mathrm{T} 2$ was present and clearly visible, i.e. at lag 8 , the distributions showed a single peak of responses at $100 \%$ visibility ( $47 \%$ of the responses in single task, $49.3 \%$ in dual task). Crucially, during the AB, we observed a mixture of those two states (see Fig. 2c). The 
percentage of responses at $100 \%$ visibility dropped then increased again with lag, in synchrony with an opposite increase and decrease of the percentage of responses at $0 \%$ visibility. At lag 3, the percentage of responses for 0 and $100 \%$ visibility were almost equal (21.7\% and 21.4\%). The ANOVA restricted to the T2 present condition revealed significant visibility $\times$ task, visibility $\times$ lag and visibility $\times$ task $\times$ lag interactions $\left(p<.005, \eta^{2}>.13\right)$.

We tested the hypothesis that responses during the $\mathrm{AB}$ are a mixture of discrete seen and not-seen states by submitting the response distribution at each lag to a linear regression, using two predictors : the response distribution observed when T2 was present ("present" predictor) and the distribution observed when T2 was absent ("absent" predictor) at lag 8 in the dual-task condition (see Fig. 2d). For all lags from 1 to 6 , this model accounted for more than $91 \%$ of the variance, with significant contributions of both predictors $(p<.005)$. In particular, the significant contribution of the "absent" predictor indicated that some of the T2-present trials were subjectively indistinguishable from T2-absent trials. The same regressions in the single-task condition revealed no significant contribution of the "absent" predictor in explaining the response distributions $(t(18)<2, p>.09)$, except for a small but significant contribution at lag $3(t(18)=3, p=.007)$. Thus, at each lag except lag 3 , the “present" predictor was sufficient to give a satisfactory model of the distribution $\left(r^{2}>90 \%\right)$.

In summary, although the scale was designed to be sensitive to continuous changes of perception, participants used it in an all-or-none fashion. The $\mathrm{AB}$ did not result in a gradual reduction of $\mathrm{T} 2$ visibility but in an increase in the proportion of trials where $\mathrm{T} 2$ was missed and had the same visibility than on absent trials. These results support the hypothesis that the $\mathrm{AB}$ consists in a stochastic all-or-none loss of conscious access to $\mathrm{T} 2$. 


\section{EXPERIMENT 2 : GRADUAL RESPONDING \\ IN A MASKING PARADIGM.}

The all-or-none response patterns observed in Experiment 1 could reflect a response bias toward both ends of the scale. Participant might have implemented the instructions as a forced-choice task between "T2 seen" and "T2 not seen", using a sharp decision criterion. Experiment 2 rules out this possibility by showing that, with the same instructions, participants spontaneously used the scale in a continuous fashion when judging the visibility of masked words of variable durations.

\section{Methods}

\section{Subjects}

Ten right-handed native French speakers (6 women and 4 men; age ranging from 21 to 25) took part in Experiment 2. All had normal or corrected-to-normal vision.

\section{Design and stimuli}

Each trial consisted in a fixation cross presented at the center of the screen for 301 or $473 \mathrm{~ms}$ with the same probability, followed by the target (number word or blank), the mask and the subjective visibility scale ( $300 \mathrm{~ms}$ after target onset). The total duration of target plus mask was fixed (300 ms). We used six different target duration : 14, 29, 43, 57, 71 and $86 \mathrm{~ms}$. The mask was created on each trial by semi-random arrangements of diamond and square shapes, covering up the central area of screen where the target appeared (approximately $4,5^{\circ} \times$ $\left.1,5^{\circ}\right)$. After a short training, each participant performed 40 trials in each presence $\times$ duration condition. 


\section{Results}

When the target was absent, duration had no effect on mean visibility $(F<1)$ : the response distribution always showed a single peak at visibility 0 . When the target was present, mean subjective visibility increased significantly with duration $(F(5,45)=182.6, p<.0001$, $\eta^{2}=.95$ ) (see Fig. 3b). Response distributions (Fig. 3c) showed a gradual displacement towards higher visibility degrees with increasing target duration, yielding a significant visibility $\times$ duration interaction $\left(F(100,900)=8.5, p<.0001, \eta^{2}=.49\right)$. At each target duration, the distribution was unimodal, responses clustering around one visibility rating, contrary to the bimodal distribution found in Experiment 1. A multiple linear regression was conducted on the various distributions using as predictors the distributions obtained in the target present and in the target absent conditions at maximal target duration $(86 \mathrm{~ms})$. The "absent" predictor did no significantly contribute to those regressions $(p \mathrm{~s}>.16)$ except for $14 \mathrm{~ms}$ duration $(t$ $(18)=10.43, p<.0001)$. Thus, contrary to what was found in the AB experiment, participants did not use the scale in an all-or-none fashion. The scale was sensitive to gradual changes in perception. The contrasting response patterns obtained in the two experiments suggest that $\mathrm{AB}$ and masking may have different underlying mechanisms. 


\section{EXPERIMENT 3 : COMBINED ALL-OR-NONE AND GRADUAL RESPONDING IN A MIXED AB-MASKING PARADIGM.}

It could still be argued that, in Experiment 1, responses were biased towards the left and right ends of the scale, whereas, with the simpler stimuli used in Experiment 2, participants succeeded in making the required subtle subjective visibility judgement. We therefore conducted another $\mathrm{AB}$ experiment in which we forced participants to use the scale gradually by manipulating the strength of $\mathrm{T} 2$ masking. In this experiment, which combined $\mathrm{AB}$ and masking, we predicted a mixture of discrete and continuous response patterns. At long T1-T2 lag, responses should shift gradually towards higher visibility with increasing T2 duration. At short lag, this distribution should split up and become bimodal, with one peak appearing at the "not-seen" end.

\section{Methods}

\section{Subjects}

Ten right-handed native French speakers ( 5 women and 5 men; age ranging from 21 to 25) took part in Experiment 3. All had normal or corrected-to-normal vision.

\section{Design and stimuli}

Each trial consisted in an RSVP sequence identical to the one used in Experiment 1 except that it ended with a 129 ms blank followed by the target (T2) and mask of Experiment 2, and the subjective scale (300 ms after T2 onset). We used six different T2 duration $(14,29,43,57,71$ and $86 \mathrm{~ms})$, two different lags (3 and 8, corresponding to SOAs of $258 \mathrm{~ms}$ and $688 \mathrm{~ms}$ respectively), and only the dual-task condition of Experiment 1 . After a 
short training, each participant performed 32 trials in each lagx 2 -duration condition for T2present and 8 trials in each lag $\times \mathrm{T} 2$-duration condition for $\mathrm{T} 2$-absent.

\section{Results}

Trials with an incorrect response to $\mathrm{T} 1$ ( $2 \%$ to $11 \%$ across participants) were discarded. When T2 was absent, duration had no effect on mean visibility $(F(5,45)<1)$ (see Fig. 3e). Mean visibility was slightly higher at lag 3 than at lag $8(F(1,9)=9.87, p=.012$, $\left.\eta^{2}=.52\right)$. There was no significant lagxduration interaction $(F(5,45)<1)$. Response distributions showed a single peak at 0 visibility. When $\mathrm{T} 2$ was present, mean visibility increased significantly with T2 duration $\left(F(5,45)=217.89, p<.0001, \eta^{2}=.96\right)$ and the interaction between duration and lag was significant $\left(F(5,45)=4.31, p=.003, \eta^{2}=.32\right)$ indicative of a significant $\mathrm{AB}$ effect. Figure $3 \mathrm{f}$ shows the corresponding response distributions : at T2 duration $=14$ and $29 \mathrm{~ms}$, the response distributions showed a peak at 0 visibility. At higher T2 duration, two groups of responses could be distinguished : a peak at 0 visibility that decreased with lag, and a peak that gradually shifted towards higher visibility with increasing $\mathrm{T} 2$ duration and thus progressively dissociated from the peak at 0 . At $\mathrm{T} 2$ duration $=57 \mathrm{~ms}$, short lag, the distribution was clearly bimodal with a peak of $15.1 \%$ of responses at $0 \%$ visibility and another around $65 \%$ visibility. At long lag, for the same T2 duration, the peak at 0 was much reduced. It resulted in a significant visibility $\times$ lag interaction at this duration $\left(F(20,180)=1.93, p=.013, \eta^{2}=.18\right)$.

Thus, the AB seemed to increase the number of "not seen" responses without affecting the gradual increase in peak visibility with increasing T2 duration. In order to test this hypothesis, for each T2 duration we conducted linear regressions of the distributions obtained at short lag using as "present" predictor the corresponding response distribution at long lag when T2 was present, and as "absent" predictor the distribution obtained at long lag when T2 
was absent and T2 duration was maximal ( $86 \mathrm{~ms})$. For all T2 durations, $r^{2}$ was above $86 \%$. At short (14 ms, $29 \mathrm{~ms})$ and long $(71 \mathrm{~ms}, 86 \mathrm{~ms})$ T2 duration, the contribution of the "absent" predictor was not significant, the "present" predictor being sufficient to model the distribution $(t(18)>4, p<.001)$. At T2 duration $=43 \mathrm{~ms}$ and $57 \mathrm{~ms}$, however, the contribution of the T2absent predictor was highly significant $(t(18)>4, p<.001)$ while the contribution of the T2present predictor remained significant $(t(18)>3, p<.006)$.

In summary, although participants used the scale gradually in response to changes in $\mathrm{T} 2$ duration, the $\mathrm{AB}$ phenomenon still yielded all-or-none response patterns. Because both effects were found within the same trials, this cannot be attributed to response bias. We conclude that, whereas reducing the duration of a masked target induces a gradual degradation of subjective perception, the $\mathrm{AB}$ deficit causes an all-or-none loss of conscious access.

\section{GENERAL DISCUSSION}

Asking participants to rate $\mathrm{T} 2$ visibility on a continuous scale allowed to examine whether the $\mathrm{AB}$ results from a continuous degradation or from an all-or-none loss of conscious access. According to the first hypothesis, the AB should have yielded unimodal and gradually shifting response distributions on the visibility scale. Our results invalidate this hypothesis and demonstrate a bimodal distribution : on some trials $\mathrm{T} 2$ is entirely invisible, and on others it is as visible as when no T1 task is required.

Because we collected T2 ratings less than 300 ms after T2 was presented, our results are unlikely to be affected by a quick forgetting of having seen T2. Rather, among the various psychological accounts of the AB phenomenon, the two-stage model (Chun \& Potter, 1995) seem to give the most satisfactory explanation to these results. According to this model, a stimulus must undergo two stages of processing in order to be correctly reported and the second stage can process only one stimulus at a time. Thus, the $\mathrm{AB}$ deficit would reflect the 
fact that, as long as the second stage is occupied by $\mathrm{T} 1$, access to this stage is denied to $\mathrm{T} 2$, which is thus susceptible to be erased by a trailing mask. However, this psychological account of the $\mathrm{AB}$ does not specify the neuronal mechanisms underlying the two stages, nor their dynamics.

A series of event related potential studies by Vogel, Luck and Shapiro (Luck, Vogel, \& Shapiro, 1996; Vogel, Luck, \& Shapiro, 1998), have shown that several stages of perceptual and semantic processing are unaffected by the AB. However, the P300 wave, which reflects the updating of information in working memory, was suppressed during the $\mathrm{AB}$. These experiments further support the two-stage model of the $\mathrm{AB}$ by showing a qualitative difference in the neural processing that takes place inside and outside the $A B$, with a preservation of a first stage of processing and a complete disappearance of a second stage of processing during the blink period.

The "neuronal global workspace" model (Dehaene et al., 1998) provides a neural account of the two stages described in psychological models of the AB. According to this theory, the first stage of processing would correspond to what has been called the "feedforward sweep" (Lamme, 2003), where a stimulus is automatically processed by a series of brain areas activated sequentially in a bottom-up manner. The second stage would correspond to top-down amplification. On conscious trials, bottom-up and top-down inputs reinforce each other until a broad network of cerebral areas becomes ignited via long-distance connections. The entry of the stimulus into this global workspace allows the maintenance of information and the flexibility of processing that characterize conscious perception. If, however, this first activation does not reach the dynamic threshold for self-amplification, activation is confined to a bottom-up transient and the stimulus cannot be consciously perceived. In this interpretation, the blink acts by cutting the top-down support for $\mathrm{T} 2$, because workspace neurons are temporarily occupied by coding T1. A neuronal network simulation of this model (Dehaene et al., 2003) was shown to be able to reproduce the results from experiment 1 , 
including the all-or-none response of workspace neurons and the influence of the lag on the proportion of seen and unseen trials.

What determines whether a stimulus reaches the threshold for conscious access? It might be small stochastic differences in the first wave of activation or even in the baseline activity preceding the stimulus. Indeed, for threshold stimuli, the BOLD signal in V1 and the P100 wave were shown to be larger on seen trials than on unseen trials (Pins \& Ffytche, 2003). Furthermore, a multiunit recording study in monkeys' primary visual cortex demonstrated that $100 \mathrm{~ms}$ prior to stimulus onset, neural response was already stronger for a subsequently reported stimulus than for a not-reported stimulus (Super, van der Togt, Spekreijse, \& Lamme, 2003). In the AB, stochastic differences in the time and effort spent to process T1 might determine the all-or-none perception of T2 (Marois, Chun, \& Gore, 2000).

We used masking as a control to demonstrate that subjects could use the visibility scale in a graded manner. The findings suggest that masking obeys a different principle of gradually increasing perception as a function of target duration. One possibility is that, as target duration increases, the bottom-up wave of activity gains strength and increasingly deeper processing stages are activated. Thus, the information that enters consciousness is increasingly richer. Indeed, subjects reported seeing increasingly more detailed aspects of the masked stimuli, from a few features to single letters, graphemes, and finally the whole word, which they traduced by continuously varying the cursor on the visibility scale. Importantly, this does not mean that, were we to test a single level of visual perception (e.g. Vernier acuity), we would not find a discontinuous threshold for perception as a function of exposure duration. Indeed, there is a suggestion of a non-linear threshold effect in figure $3 \mathrm{c}$, where cursor position seems to jump as target duration changes from $29 \mathrm{~ms}$ to $43 \mathrm{~ms}$. Further research is needed to explore this effect with a higher temporal resolution.

More generally, our work suggests that conscious access is characterized by non-linear dynamical phenomena, which might ultimately be described mathematically using catastrophe 
theory (Saunders, 1980; Thom, 1972). The visibility scale methodology may provide an important tool with which to trace the "bifurcation diagrams" of subjective perception. 
Acknowledgments_we thank J-P. Changeux, L. Naccache, V. Izard and E. Spelke for useful comments. This study was supported by INSERM and a centennial fellowship from the Mc Donnell Foundation to S. Dehaene. 


\section{REFERENCES}

Baars, B. J. (1989). A cognitive theory of consciousness. Cambridge, Mass.: Cambridge University Press.

Baars, B. J. (1997). In the theater of consciousness: Oxford University Press.

Bar, M., Tootell, R. B., Schacter, D. L., Greve, D. N., Fischl, B., Mendola, J. D., et al. (2001). Cortical mechanisms specific to explicit visual object recognition. Neuron, 29(2), 529535.

Broadbent, D. E., \& Broadbent, M. H. (1987). From detection to identification: response to multiple targets in rapid serial visual presentation. Percept Psychophys, 42(2), $105-$ 113.

Chun, M. M., \& Potter, M. C. (1995). A two-stage model for multiple target detection in rapid serial visual presentation. J Exp Psychol Hum Percept Perform, 21(1), 109-127.

Dehaene, S., Kerszberg, M., \& Changeux, J. P. (1998). A neuronal model of a global workspace in effortful cognitive tasks. Proc Natl Acad Sci U S A, 95(24), 1452914534.

Dehaene, S., \& Naccache, L. (2001). Towards a cognitive neuroscience of consciousness: basic evidence and a workspace framework. Cognition, 79(1-2), 1-37.

Dehaene, S., Naccache, L., Cohen, L., Bihan, D. L., Mangin, J. F., Poline, J. B., et al. (2001). Cerebral mechanisms of word masking and unconscious repetition priming. Nat Neurosci, 4(7), 752-758.

Dehaene, S., Sergent, C., \& Changeux, J. P. (2003). A neuronal network model linking subjective reports and objective physiological data during conscious perception. Proc Natl Acad Sci U S A. 
Di Lollo, V., Enns, J. T., \& Rensink, R. A. (2000). Competition for consciousness among visual events: the psychophysics of reentrant visual processes. J Exp Psychol Gen, 129 (4), 481-507.

Farah, M. J. (2000). The cognitive neuroscience of vision. Oxford: Blackwell.

Grill-Spector, K., Kushnir, T., Hendler, T., \& Malach, R. (2000). The dynamics of objectselective activation correlate with recognition performance in humans. Nat Neurosci, $3(8), 837-843$.

Jolicoeur, P. (1998). Modulation of the attentional blink by on-line response selection: evidence from speeded and unspeeded task1 decisions. Mem Cognit, 26(5), 10141032.

Kanwisher, N. (2001). Neural events and perceptual awareness. Cognition, 79(1-2), 89-113. Lamme, V. A. (2003). Why visual attention and awareness are different. Trends Cogn Sci, 7 (1), 12-18.

Lamme, V. A., \& Roelfsema, P. R. (2000). The distinct modes of vision offered by feedforward and recurrent processing. Trends Neurosci, 23(11), 571-579.

Lamme, V. A., Super, H., Landman, R., Roelfsema, P. R., \& Spekreijse, H. (2000). The role of primary visual cortex (V1) in visual awareness. Vision Res, 40(10-12), 1507-1521.

Luck, S. J., Vogel, E. K., \& Shapiro, K. L. (1996). Word meanings can be accessed but not reported during the attentional blink. Nature, 383(6601), 616-618.

Marois, R., Chun, M. M., \& Gore, J. C. (2000). Neural correlates of the attentional blink. Neuron, 28(1), 299-308.

Massaro, D. W., \& Cohen, M. M. (1983). Categorical or continuous speech perception: a new test. Speech communication, 2, 15-35.

Moutoussis, K., \& Zeki, S. (2002). The relationship between cortical activation and perception investigated with invisible stimuli. Proc Natl Acad Sci U S A, 99(14), 9527-9532. 
Pallier, C., Dupoux, E., \& Jeannin, X. (1997). Expe5: an expandable programming language for on-line psychological experiments. Behavior Research, Methods, Instruments and Computers, 29, 322-327.

Pins, D., \& Ffytche, D. (2003). The neural correlates of conscious vision. Cereb Cortex, 13 (5), 461-474.

Raymond, J. E., Shapiro, K. L., \& Arnell, K. M. (1992). Temporary suppression of visual processing in an RSVP task: an attentional blink? J Exp Psychol Hum Percept Perform, 18(3), 849-860.

Saunders, P. T. (1980). An introduction to catastrophe theory. Cambridge, Mass.: Cambridge University Press.

Super, H., Spekreijse, H., \& Lamme, V. A. (2001). Two distinct modes of sensory processing observed in monkey primary visual cortex (V1). Nat Neurosci, 4(3), 304-310.

Super, H., van der Togt, C., Spekreijse, H., \& Lamme, V. A. (2003). Internal state of monkey primary visual cortex (V1) predicts figure-ground perception. J Neurosci, 23(8), 34073414.

Thom, R. (1972). Stabilité structurelle et Morphogénèse. Paris: InterEditions.

Vogel, E. K., Luck, S. J., \& Shapiro, K. L. (1998). Electrophysiological evidence for a postperceptual locus of suppression during the attentional blink. J Exp Psychol Hum Percept Perform, 24(6), 1656-1674. 


\section{Figure Captions}

Fig. 1. Prediction of a discontinuous transition in non-linear dynamical systems with selfamplification. Left : generic curve describing activation in a non-linear self-amplifying system (y axis) as a function of a control parameter ( $\mathrm{x}$ axis) which represents the combined influences of intensity and duration of the current stimulus as well as inhibitory influences for other concurrently processed stimuli. Right : predicted response distributions on a subjective visibility scale. Progressively decreasing the control parameter from an above-threshold value ( $\mathrm{T}$ : threshold) initially leads to a gradual decrease in global activation and thus in subjective visibility. At threshold, however, there is a discontinuous jump to a lower level of activation, corresponding to lack of sustained activation and therefore, according to the global neuronal workspace model, an absence of conscious perception. There is a range of control parameter values (thick gray segment) within which both high and low states of activation coexist.

Fig. 2. Experiment 1. A : design. Each trial consisted in a rapid serial presentation of letter strings including two targets (T1 and T2) separated by a variable lag, followed by a question on T2 (Q2 : visibility scale) and, in the dual task condition, a question on T1(Q1). B : mean subjective visibility obtained at each tested lag for the various T2-presencextask conditions followed a classical $\mathrm{AB}$ pattern. $\mathbf{C}$ : the graphs show response distributions on the scale in the T2 present, dual task condition. Above : histogram representation; below : contour plot. D : A combination of the response distributions observed for T2 absent and T2 present at lag 8 predicted response distributions at each lag that accounted for more than $91 \%$ of the variance in the observed distributions in $\mathrm{C}$. 
Fig. 3. Experiment 2 (A, B and C) and Experiment 3 (D, E and F). Experiment 2 was a masking experiment in which the target duration was varied (A). There was a fixed $300 \mathrm{~ms}$ SOA between the target and the question (Q : visibility scale). B : the graph shows the mean subjective visibility obtained at each tested target duration in the target present and target absent conditions. $\mathbf{C}$ : the graphs show response distributions on the scale in the target present condition. Above : histogram representation; below : contour plot. Experiment 3 was a mixture of Experiments 1 and 2 where T1-T2 lag and T2 duration were varied (D). E : the graph shows the mean subjective visibility obtained at each T2 duration in the various $\mathrm{T} 2$ presencexlag conditions. $\mathbf{F}$ : the graphs show response distributions on the scale in the T2 present condition at lag 3 (left) and lag 8 (right). Above : histogram representations; below : contour plots. 
Figure 1

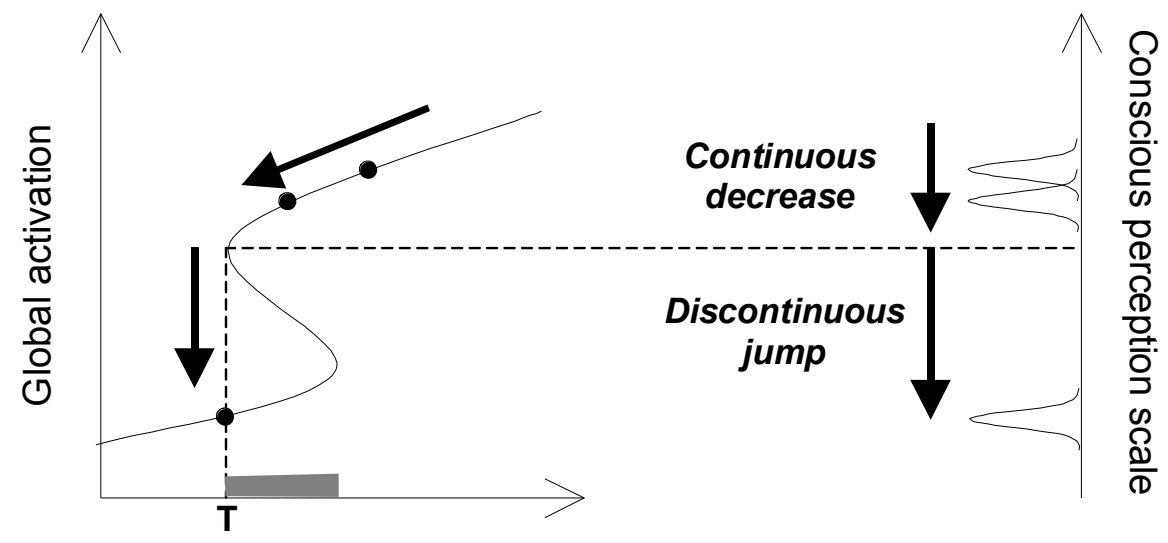

Control parameter

eg. lateral inhibition from active representations 
Figure 2

A

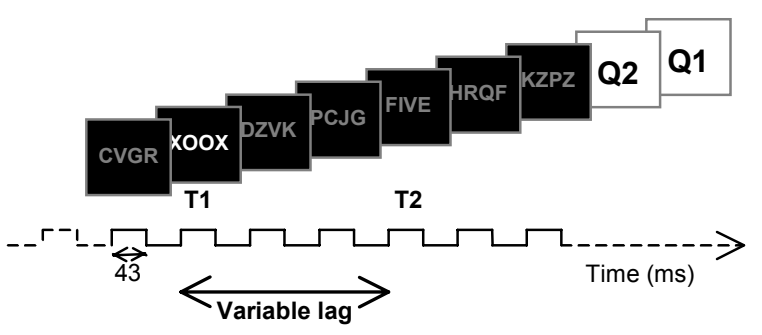

B

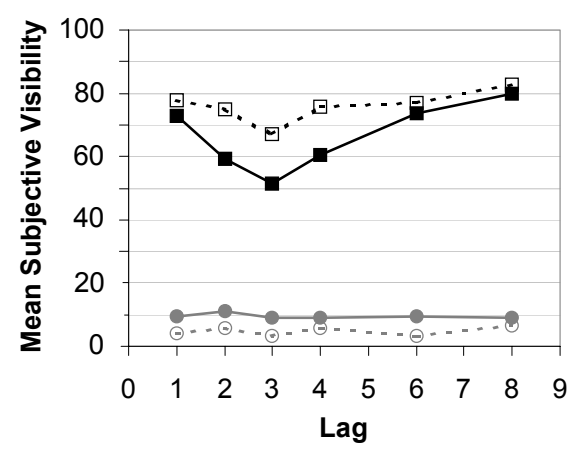

$\rightarrow-T 2$ present, dual task - - $\square-$ T2 present, single task - - T2 absent, dual task $\quad-\odot-$ T2 absent, single task

D

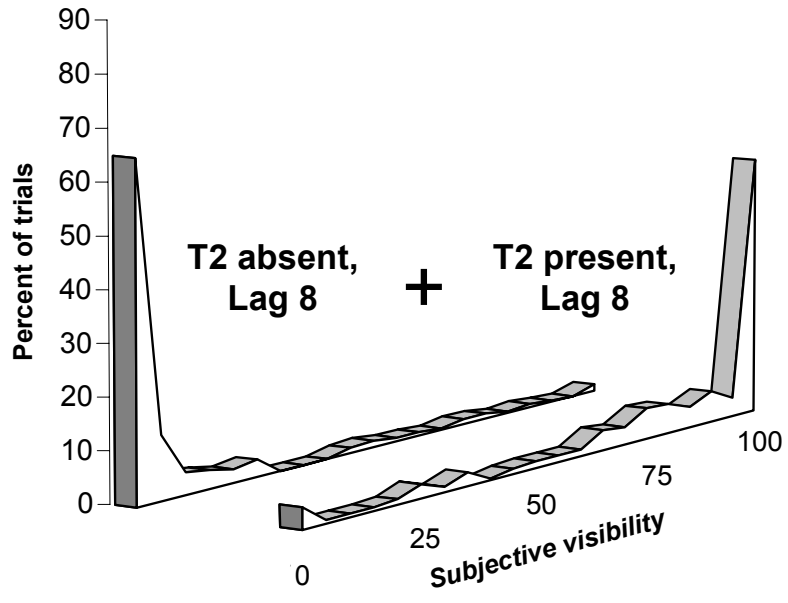

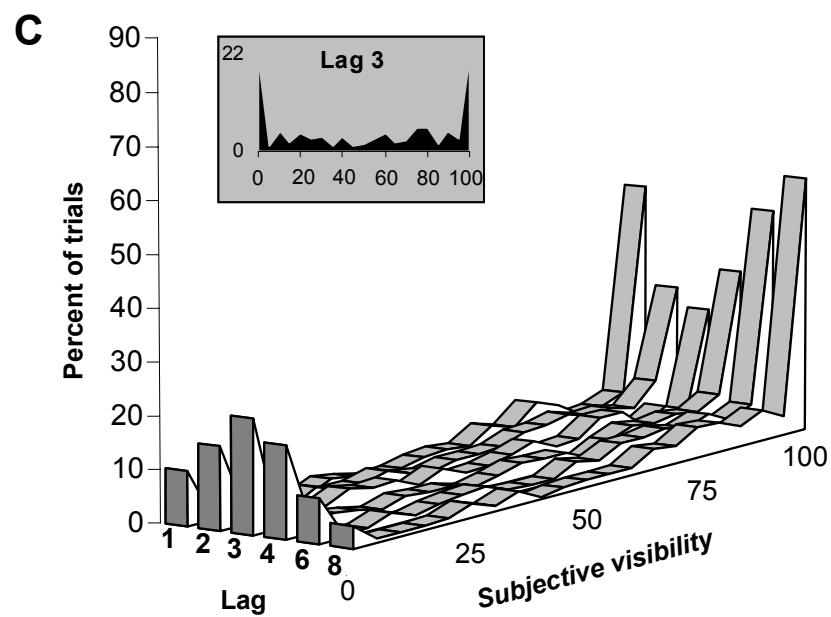
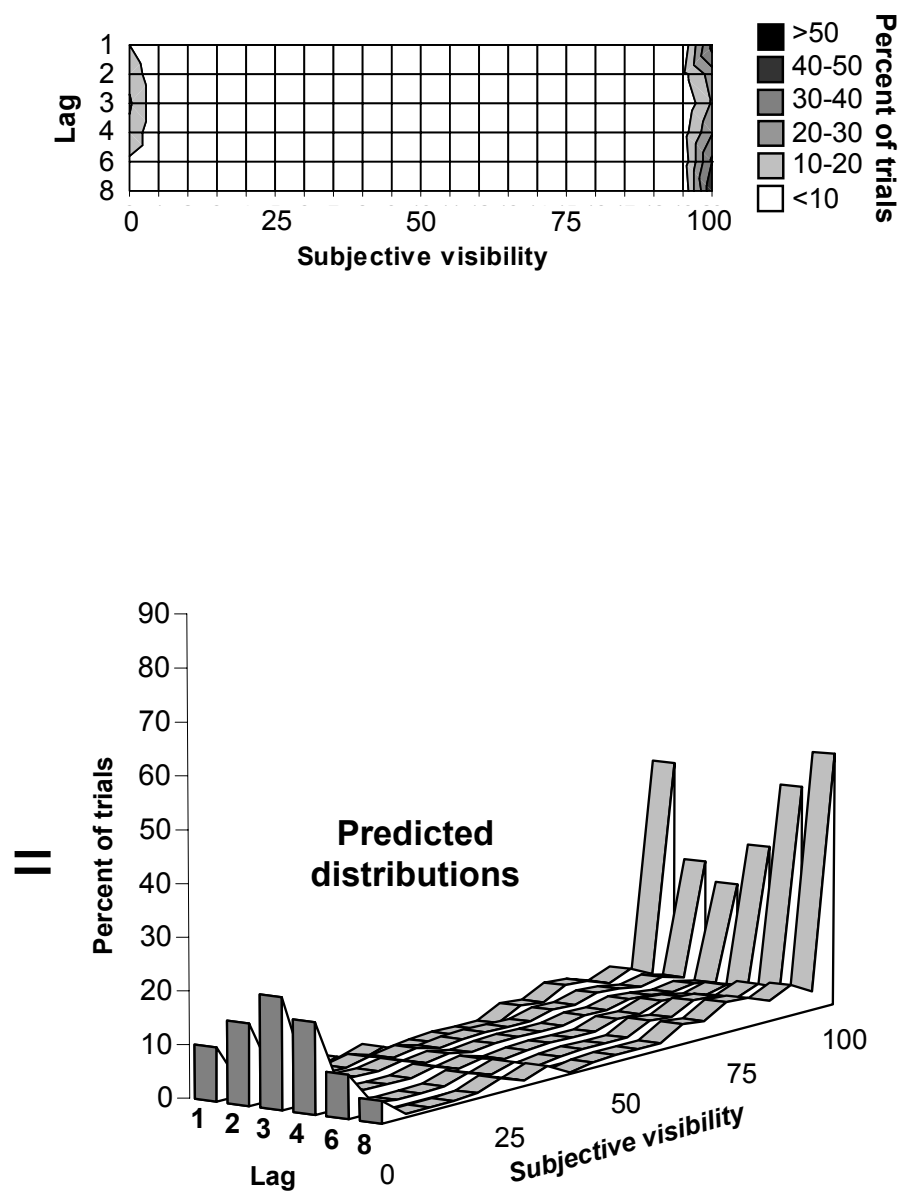
Figure 3

A

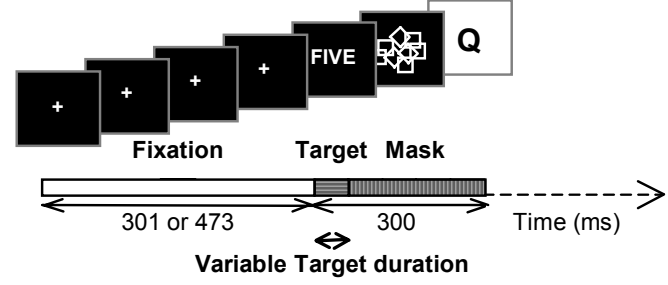

B

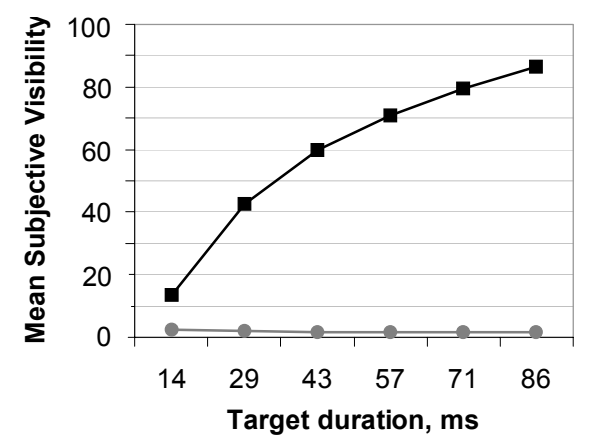

$\rightarrow$-Target present $\quad \rightarrow-$ Target absent

D
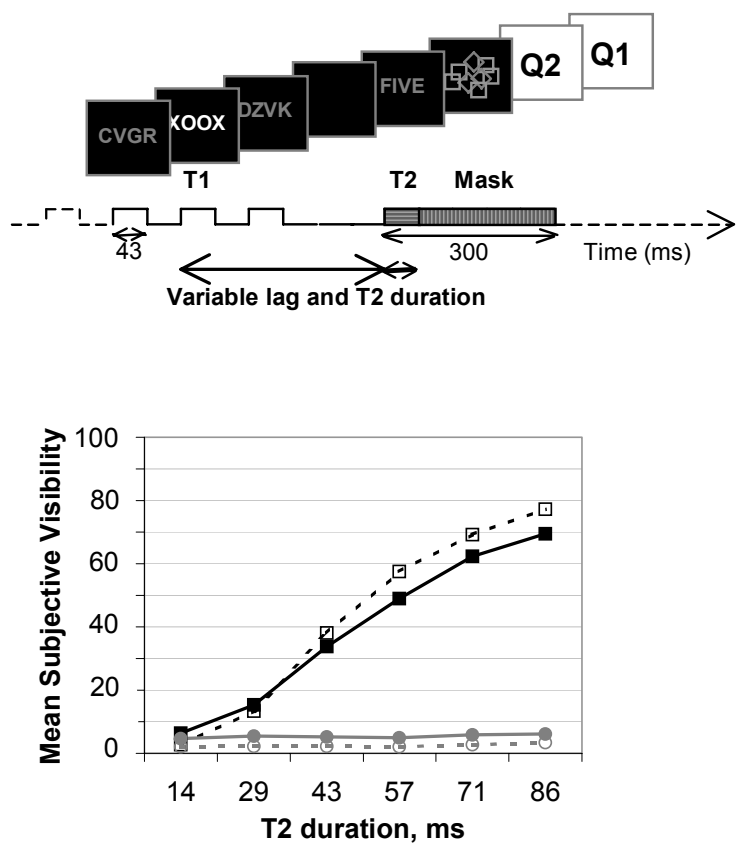

$\rightarrow$ T2 present, lag 3

- - T2 present, lag 8

T2 absent, lag 3

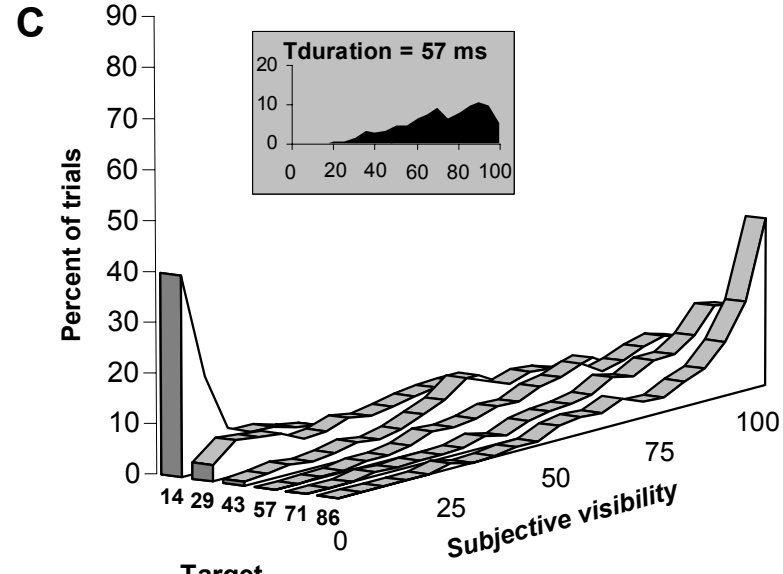

Target

duration

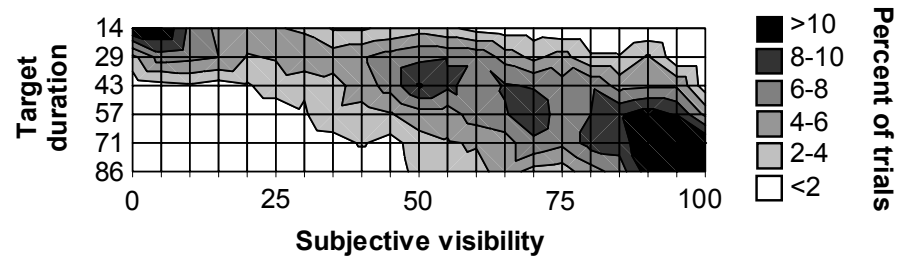

$\mathbf{F}$
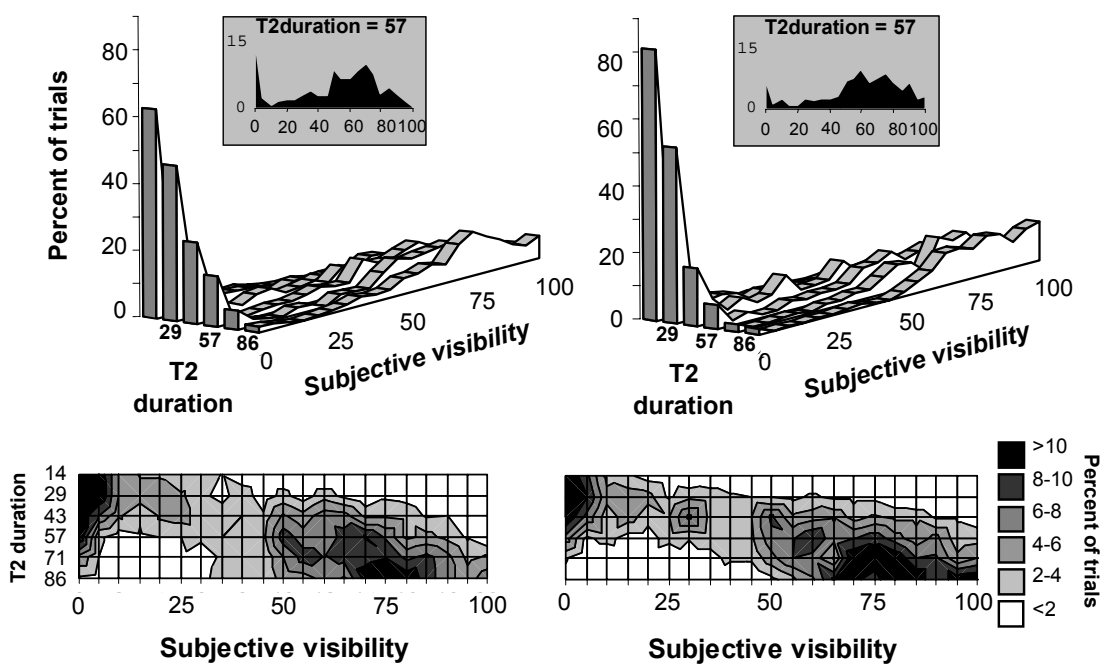